\title{
Optimization Model of Unmanned Aerial Vehicle Distribution Path with Integrated Loading and Unloading
}

\author{
Lei WU a , Ming WEI ${ }^{\mathrm{b} 1}$, Xiang CHEN ${ }^{\mathrm{a}}$ \\ a Information Science and Technology College, Nantong University, Nantong 2260191, \\ China \\ b Air Traffic Management College, Civil Aviation University of China, Tianjin 300300, \\ China
}

\begin{abstract}
Unmanned aerial vehicle (UAV) delivery has the advantages of small size, high speed, and low cost. A new drone delivery path optimization model with loading and unloading integration is proposed in this study to make full use of UAV(drone) delivery by improving its efficiency. The model considers drone range constraints and loading capacity limitations, analyzes the start and end points of multiple orders, assigns orders to drones from the optimal distribution centers, calculating the order and time to visit all sets and delivery points, and pursuing the least transportation mileage. The ant colony optimization (ACO) algorithm is adopted to solve the problem in two stages. In the first stage, construction rules and pheromones of the solution are defined, and the orders to the UAVs are assigned. In the second stage, by adding constraints to the ACO algorithm, the sequence order of the UAVs visiting the set and delivery points is determined to obtain the optimal path. Finally, a GIS-based delivery platform is developed using Java Development Kit, which is used to produce the optimal scheduling scheme for an example case. A sensitivity analysis of the model parameters is conducted $t$, which proves the proposed model effectiveness.
\end{abstract}

Keywords. UAV distribution path, Integrated loading and unloading, Ant colony algorithm, Scheduling platform development

\section{Introduction}

Unmanned aerial vehicle (UAV) or drone delivery is a new industry that has developed rapidly in recent years. In the context of the global epidemic outbreak in 2020, drone delivery has gained even more attention. Making full use of drone delivery to deliver items to customers efficiently and safely has become a major problem. Compared with conventional vehicle (truck) delivery, drone delivery is not restricted by the road network traffic operation situation of space and time, which can solve the 'last mile' delivery efficiency, cost, safety, and other issues. There are differences between truck and drone delivery models. Thus, despite some commonalities between traditional vehicle and UAV delivery path theories, the available findings and approaches concerning the former (truck delivery) cannot be

\footnotetext{
${ }^{1}$ Corresponding Author: Ming WEI, Air Traffic Management College, Civil Aviation University of China, Tianjin 300300, China; Email: mingtian911@163.com
} 
directly applied to the UAV delivery problem. Therefore, the UAV delivery path optimization problem has attracted extensive attention from scholars worldwide.

In 2015, Murray and Chu [1] proposed the flying sidekick traveling salesman problem (FSTSP) as a new variant of the TSP problem for solving the path planning of cooperative truck-and-drone transportation. Due to the problem complexity, they considered only one truck and one drone, and the drone carried only a single package for delivery. Then, Agatz [2] formulated the traveling salesman problem with drone (TSP-D) problem and solved it using dynamic programming methods. Like the FSTSP problem, TSPD considered the use of a single truck and a single drone. Ha et al. [3] proposed two heuristics for TSPD to optimize the operation cost. Marinelli et al. [4] studied the TSP-D where the drone take-off and return locations could be different and designed a so-called greedy algorithm. Wang et al. [5] reported that the time of the joint truck-and-drone delivery was less than that of truck delivery, even in the worst case. Ferrandez et al. [6] assumed that all goods/parcels were delivered by drones only, while trucks were used as movable delivery points carrying drones, packages, and charging devices. The K-means clustering algorithm was used to determine the truck delivery points, and then a genetic algorithm was applied to optimize the truck. Chang and Lee [7] used the K-means clustering to optimize the truck delivery path by minimizing the delivery time after classifying the customers.

In all the above studies, it was assumed that the UAV could only carry one package at a time for a single-point delivery. However, single-point delivery will increase the flight distance between UAVs' supply and demand points, causing a waste of UAV flight resources. While relevant studies have shown that full operation of multiple UAVs could effectively improve distribution efficiency [8,9], some researchers $[10,11,12]$ have considered that UAVs could carry multiple parcels at a time. Still, these studies did not consider the simultaneous UAV pick-up and delivery, which would envision that upon completing the delivery task, each UAV could go to another location to pick up the parcels to be delivered before completing the subsequent task. In the UAV multipoint delivery operation, route planning is a key factor that directly affects the efficiency of delivery. Kitjacharoenchai et al. [13] proposed a mixed integer planning method for UAV multipoint delivery route search to reduce truck-and-UAV delivery operation time. Salama et al. [14] used an unsupervised machine-learning heuristic algorithm to study UAV's warehouse's location' points and UAV multipoint delivery routes.

The existing approaches have the following two main shortcomings: (1) they involve separated loading and unloading and separated analyses of the UAV collection and delivery paths; (2) they ignore the presence of no-fly zones in the UAV delivery paths.

To mitigate the above shortcomings, this paper formulates the integrated loading and unloading UAV delivery path optimization problem with the account of no-fly zones, as well as applies the ant colony optimization (ACO) algorithm to solve this problem. When several customers place orders involving the start and end points and the number of parcels, the orders are assigned to drones. Such constraints as no-fly zones and drone performance parameters are considered to determine from which distribution center the drones should depart. The order and time to visit all sets and delivery points are calculated to pursue the least distribution cost. Finally, a GIS-based distribution platform developed in Java script is applied to a simple delivery task example to yield the optimal scheduling scheme. Finally, the parameter sensitivity analysis of the model is performed to verify its effectiveness and feasibility. 


\section{Problem Formulation}

The global problem studied in this paper has several constraints and separate subtasks. There are one or more distribution centers and several customer orders, each with information on the origin and destination (collection and delivery points) and the number of parcels to be delivered (cargo). If there is a no-fly zone between any set and delivery points, no direct UAV flight is allowed. Realistic constraints such as no-fly zone, maximum mileage, and maximum load weight of the UAV are considered. Using the drone delivery platform, one has to collect customer order information, assign orders to drones, determine any drone from the best distribution center, calculate the order and time to visit all sets and delivery points and pursue the least distance of drone delivery [10]. When a drone visits the delivery point of a customer order, it must ensure that there is a sufficient number of parcels on the drone to be unloaded, i.e., the drone needs to visit the collection point of that customer order in advance and pick up a sufficient number of parcels. For the spatio-temporal distribution of customer order demands, it is urgent to reveal the intrinsic relationship between the number of drones in the distribution center, the delivery path, and the transportation cost to optimize the resource allocation and pursue the highest system efficiency. Without loss of generality, the following assumptions can be used to make the model more realistic:

(1) Through the online website or mobile client, collect the starting and ending points of all customer orders and the number of goods, distribute the goods (parcels) according to the unified time window, and ignore the impact of the customer's personalized time window on the distribution plan.

(2) Considering the no-fly zone effect on the flight distance between any collection and delivery points, the actual distance can be obtained through available digital maps (e.g., Baidu maps in China or Google maps elsewhere), ignoring the takeoff or landing distance.

(3) The effect of the waiting time of UAV in the collection and delivery points on the distribution plan can be ignored.

To illustrate the mechanism of this problem's solution, two alternative scheduling schemes, namely LBU (loading before unloading) and ILU (integrated loading and unloading), are applied to a simple case. The case comprises one distribution center (D) and four customer orders with collection and delivery points of (S1, E1), (S2, E2), (S3, E3), and (S4, E4), with respective parcel (cargo) weights of 2, 3, 2, and $1 \mathrm{~kg}$. The delivery routes of two available drones, UAV1 and UAV 2, are D-S1-S2-E2-E1-D and D-S3-S4-E4-E3-D for the ILU mode versus D-S1-E1-S2-E2-D and D-S3-E3-S4-E4-D for LBU mode. Combined with the air traffic network travel distance, the objective function can be calculated accordingly.

The definitions and symbols of all parameters and variables used in this study are explained as follow.

In terms of Index, i and j represent Pick-up or delivery index, k represents UAV index,o represents Order index, D represents Set of distribution centers, $N$ represents Set of pick-up or delivery points, K represents Set of UAV. In terms of Parameter, $w_{i}^{k}$ represents Weight of the UAV k at pick-up or delivery point $\mathrm{i}, \mathrm{d}_{\mathrm{ij}}$ represents Flight distance between pick-up or delivery points $\mathrm{i}$ and $\mathrm{j}, \mathrm{q}_{\mathrm{o}}$ represents Number of parcels at the order $\mathrm{o}, \mathrm{u}_{\mathrm{i}}^{\mathrm{o}}$ represents The pick-up point $\mathrm{i}$ of order $\mathrm{o}, \mathrm{v}_{\mathrm{j}}^{\mathrm{o}}$ represents The delivery point $\mathrm{j}$ of order o, Q represents Maximum cargo load, L represents Maximum mileage, 
$M$ represents Fixed large constant. In terms of Decision variable, $z_{i}^{k}$ represents Whether the UAV $\mathrm{k}$ visits the pick-up or delivery point $\mathrm{i}, \mathrm{x}_{\mathrm{ij}}^{\mathrm{k}}$ represents Whether the UAV k sequentially visits the pick-up or delivery points $\mathrm{i}$ and $\mathrm{j}, \mathrm{y}_{\mathrm{o}}^{\mathrm{k}}$ represents Whether the customer order $\mathrm{o}$ is assigned to the UAV k, $\mathrm{s}_{\mathrm{ij}}$ represents Whether there is a no-fly zone between pick-up or delivery points $\mathrm{i}$ and $\mathrm{j}$.

The mathematical model of the problem solution, using the relevant variables and symbols, can be described as follows.

In the proposed model, the objective function of the problem, which seeks to minimize the total travel distance, is derived via equation (1):

$$
\min \mathrm{f}=\sum_{\forall \mathrm{k} \in \mathrm{K}} \sum_{\forall \mathrm{i}, \mathrm{j} \in \text { DuN }} \mathrm{d}_{\mathrm{ij}} \cdot \mathrm{x}_{\mathrm{ij}}^{\mathrm{k}}
$$

The problem's constraints are formulated via equations (2)-(11) as follows.

Equation (2) implies that each customer order is uniquely assigned to a drone:

$$
\sum_{\forall \mathrm{k} \in \mathrm{K}} \mathrm{y}_{\mathrm{o}}^{\mathrm{k}}=1 \quad \forall \mathrm{o} \in \mathrm{O}
$$

Equations (3) and (4) indicate that the origin and destination of each order are ensured to be visited by a drone:

$$
\begin{aligned}
& u_{i}^{o}+\left(1-y_{o}^{k}\right) \cdot M \leq z_{i}^{k} \forall o \in 0, \forall i \in N, \forall k \in K \\
& v_{j}^{o}+\left(1-y_{o}^{k}\right) \cdot M \leq z_{j}^{k} \forall o \in 0, \forall j \in N, \forall k \in K
\end{aligned}
$$

Equation (5) indicates that the set, delivery point visited by a drone is in the path:

$2 \mathrm{x}_{\mathrm{ij}}^{\mathrm{k}} \leq \mathrm{z}_{\mathrm{i}}^{\mathrm{k}}+\mathrm{z}_{\mathrm{j}}^{\mathrm{k}} \forall \mathrm{i}, \mathrm{j} \in \mathrm{N}, \forall \mathrm{k} \in \mathrm{K}$

Equation (6) indicates that each drone departs from the distribution center after completing the task and finally returns to the distribution center:

$$
\sum_{\forall j \in D \cup N} x_{i j}^{k}=\sum_{\forall j \in D \cup N} x_{j i}^{k}=z_{i}^{k} \forall i \in N, \forall k \in K
$$

Equations (7) and (8) indicate the load variation relationship between the UAV's adjacent collection and delivery points:

$$
\begin{array}{r}
w_{i}^{k}+\sum_{\forall o \in O} y_{o}^{k} \cdot u_{i}^{o} \cdot q_{o}-\sum_{\forall o \in o} y_{o}^{k} \cdot v_{i}^{o} \cdot q_{o}+\left(1-x_{i j}^{k}\right) \cdot M \leq w_{j}^{k} \forall i, j \in N, \forall k \in K(7) \\
w_{i}^{k}+\sum_{\forall o \in O} y_{o}^{k} \cdot u_{i}^{o} \cdot q_{o}-\sum_{\forall o \in O} y_{o}^{k} \cdot v_{i}^{o} \cdot q_{o}+\left(1-x_{i j}^{k}\right) \cdot M \geq w_{j}^{k} \forall i, j \in N, \forall k \in K(8)
\end{array}
$$

Equation (9) indicates that the UAV visits the delivery points to ensure that the cargo is unloaded, while the maximum cargo weight is not exceeded at the collection point:

$0 \leq \mathrm{w}_{\mathrm{i}}^{\mathrm{k}} \leq \mathrm{Q} \forall \mathrm{i} \in \mathrm{N}, \forall \mathrm{k} \in \mathrm{K}$ limit:

Equation (10) provides that the UAV's travel mileage does not exceed its upper

$\sum_{\forall i, j \in D \cup N} d_{i j} \cdot x_{i j}^{k} \leq L \quad \forall k \in K$

Equation (11) implies that no UAV is allowed to pass through the no-fly zone:

$$
s_{\mathrm{ij}} \leq \mathrm{x}_{\mathrm{ij}}^{\mathrm{k}}
$$

\section{The Ant Colony Optimization Heuristic Algorithm}

In 1991, Dorigo and Stützle introduced the ant colony optimization (ACO) algorithm[15,16]. It has been widely used in the field of vehicle routing problems. According to the relevant problem characteristics, the three decision variables $z_{i}^{k}, x_{i j}^{k}$, and $y_{o}^{k}$ are involved, where $y_{o}^{k}$ determines $z_{i}^{k}$ and $x_{i j}^{k}$. However, the problem formulated in this study is an NP-hard problem, i.e., its large-scale case cannot be solved by an exact algorithm, while a non-deterministic Turing machine can solve it in 
polynomial time. Given this, we designed an ACO-based heuristic algorithm for minimizing the delivery distance. It should assign orders to UAVs in the first stage and determine the set of orders, and the order of delivery points to be visited by UAVs by making constraints on the "ant pheromone-based" pathfinding' in the second stage. The latter constraints require that there should be a sufficient number of parcels available to be unloaded when UAVs visit the delivery points. The original ACO algorithm is refined by defining the corresponding solution construction rules, pheromones, heuristic data, and selection probabilities. The specific algorithm solution process is further described and discussed in detail.

\subsection{Deconstruction rules}

Within the ACO approach framework, each UAV (drone) is simulated by a pheromone-producing ant. In the initial stage, any ant starts from a certain distribution center. When the particular ant is located at the collection point $i\left(u_{i}^{o}\right)$ of a certain order $o(\forall o \in O)$, it will find the feasible UAV sets allowed that it is allowed to access. It selects a UAV $k$ to execute the order $o\left(y_{o}^{k}\right)$ with a certain probability and adds its delivery point $j\left(v_{j}^{o}\right)$ to the alternative path set of the UAV. If allowed $\neq \varnothing$, it sends a new UAV to execute the order to establish the feasible solution continuously. Therefore, the two stages of the deconstruction process are as follows:

\section{Stage 1: Order matching with UAV}

Step 1: Randomly select an order $\forall 0 \in 0$.

Step 2: When the ant is located in the collection point of an order $o(\forall 0 \in 0)$, it finds the accessible, feasible UAV set allowed according to the constraints described by equations (9) and (10).

Step 3: To get $y_{0}^{k}$ means to select a UAV k from allowed to execute order o with a certain probability.

Step 4: When $0=0-\{0\}$, if $0 \neq \emptyset$, go to Step 1. Otherwise, the Stage 1 algorithm is terminated, and the

\section{Stage 2: Path Planning of UAV}

Step 1: When the ant is located at the delivery point $\mathrm{j}$ of an order $\mathrm{o}(\forall \mathrm{O} \in 0)$, if the order has been allocated, it will be transferred to Step 2. Otherwise (if no order has been allocated), it will be transferred to Step 3 .

Step 2: Check the execution UAV k of the order o, determine the last visited set and the delivery point $\mathrm{i}\left(\mathrm{z}_{\mathrm{i}}^{\mathrm{k}}\right)$, and add them to the path $\mathrm{x}_{\mathrm{ij}}^{\mathrm{k}}$, that is, go to Step 4 .

Step 3: Remove the delivery point $\mathrm{j}$ of the current order $\mathrm{o}(\forall \mathrm{O} \in \mathrm{O})$ from the current path of the ant.

Step 4: The ant randomly accesses the collection point $\mathrm{i}$ or delivery point $\mathrm{j}$ of a new order $o^{\prime}\left(\forall o^{\prime} \in 0\right)$ again.

\subsection{The algorithm flow}

Step 1: Initialize the parameters such as the maximum number of iterations $\mathrm{nc}_{\max }$, pheromoneaand heuristic information $\beta$. Set the UAV performance and order data to $t$ $=0$.

Step 2: Let $\mathrm{M}$ ants be randomly located in the distribution center, and let $\mathrm{m}=1$. 
Step 3: Each ant selects the next vertex $\mathrm{j}$ according to the corresponding $\mathrm{p}_{\mathrm{ij}}^{\mathrm{m}}$ in different stages via equation (12) and puts $\mathrm{j}$ in the current solution set until all the order sets and delivery points are traversed.

$$
\mathrm{p}_{\mathrm{ij}}^{\mathrm{m}}=\left\{\begin{array}{l}
\tau_{\mathrm{ij}}^{\alpha}(\mathrm{t}) \eta_{\mathrm{ij}}^{\beta} / \sum_{\mathrm{l} \in \text { all }} \tau_{\mathrm{ij}}^{\alpha}(\mathrm{t}) \eta_{\mathrm{ij}}^{\beta}, \text { others } \\
\underset{\mathrm{l} \in \text { all }}{\operatorname{argmax}}\left\{\tau_{\mathrm{ij}} \eta_{\mathrm{ij}}^{\beta}\right\}, \text { if rand } \leq \mathrm{g}_{\mathrm{o}}
\end{array}\right.
$$

To accelerate the convergence of the initial algorithm, ant $\mathrm{m}$ tends to select the edge calculated according to $\underset{\mathrm{l} \in \mathrm{all}}{\operatorname{argmax}}\left\{\tau_{\mathrm{ij}} \eta_{\mathrm{ij}}^{\beta}\right\}$ with a certain probability $\mathrm{g}_{\mathrm{o}}$. In equation (12), the heuristic data $\eta_{i j}=1 / d_{i j}$ represent the cost of UAV's sequential access set, delivery points $i$ and $j$; the pheromone $\tau_{i j}$ is the expectation of UAV's sequential access set, delivery points $\mathrm{i}$ and $\mathrm{j}$; while all correspond to the delivery points that have not visited the order or the collection points that have visited the order.

Step 4: Calculate the objective function of the solution constructed by the ant, perform a local update of the pheromone via equation (13) and record the local optimal solution $m=m+1$, if $m \leq M$, then go to Step 3 .

$$
\tau_{i j}(t+1)=\left(1-\varepsilon\left(\tau_{i j}(t)\right) \tau_{i j}(t)+\varepsilon\left(\tau_{i j}(t)\right) \cdot Q / f^{n n}\right.
$$

Step 5: Perform a global update of pheromone via equation (14):

$$
\tau_{i j}(t+1)=\left(1-\gamma\left(\tau_{i j}(t)\right) \tau_{i j}(t)+\gamma\left(\tau_{i j}(t)\right) \cdot Q / f^{b s}\right.
$$

In equations (13) and (14), parameters $\varepsilon\left(\tau_{i j}(t)\right)$, and $\gamma\left(\tau_{i j}(t)\right)$ are positive proportional functions located at $(0,1)$ with $\tau_{i j}(t)$ as the independent variable; $f^{n n}$ and $\mathrm{f}^{\mathrm{bs}}$ are the optimal solutions searched by the nearest neighbor method and ACO algorithm at present, while $Q$ is a constant.

Step 6: When $\mathrm{t}=\mathrm{t}+1$, if $\mathrm{t} \leq \mathrm{nc}_{\max }$, go to Step 2 .

\section{Example Analysis}

\subsection{Test environment and example introduction}

To verify the model feasibility and effectiveness, a GIS-based distribution platform was elaborated in the Java Development Kit (JDK 1.8), with the main functions, including order management, traffic network management, UAV management, parameter setting, path optimization, and visualization.

Taking the epidemic emergency supply distribution as an example, three distribution centers (D1-D3), five collection points (S1-S5), 29 delivery points (E1E29), and 30 customer order data were added to this platform. A certain type of UAV with a maximum mileage of $50 \mathrm{~km}$ and a maximum cargo weight of $10 \mathrm{~kg}$ was selected. The ACO algorithm basic parameters were taken from [15], including the maximum number of iterations of 500 , the number of ants of 100 , the pheromone importance factor $\alpha=1$, and the heuristic function importance factor $\beta=4$.

\subsection{Analysis of experimental results}

Comparing the optimal dispatching plans of the ILU and LBU modes listed in Table 1, it was found that four UAVs in total were needed to complete 30 orders. 
(1) The total delivery mileage in the ILU mode was $34.1 \mathrm{~km}$, and two UAVs were needed in D3, one UAV in D1, and one UAV in D2.

(2) The total delivery mileage in the LBU mode was $36.3 \mathrm{~km}$, and two UAVs were needed in D3, one UAV in D1 and one UAV in D2.

The experimental results show that the ILU mode implied less mileage than the LBU one (34.1 vs $36.3 \mathrm{~km}$ ) with the same number of drones (4) under the constraint of the maximum operating mileage $(50 \mathrm{~km})$ and the maximum cargo weight $(10 \mathrm{~kg})$ of each drone.

Table 1. The delivery plan optimization in the ILU and LBU modes

\begin{tabular}{|c|c|c|c|c|c|c|}
\hline \multirow[b]{2}{*}{$\begin{array}{l}\text { UAV } \\
\text { No. }\end{array}$} & \multicolumn{3}{|l|}{ Integrated loading and unloading (ILU) } & \multicolumn{3}{|c|}{ Loading before unloading (LBU) } \\
\hline & Path & $\begin{array}{l}\text { Number } \\
\text { of } \\
\text { orders }\end{array}$ & $\begin{array}{l}\text { Mileage } \\
(\mathbf{k m})\end{array}$ & Path & $\begin{array}{l}\text { Number } \\
\text { of } \\
\text { orders }\end{array}$ & $\begin{array}{l}\text { Mileage } \\
(\mathbf{k m})\end{array}$ \\
\hline 1 & $\begin{array}{l}\text { D2(0)-S3(4)-S6(10)-E9(8)-E5(6)- } \\
\text { E2(5)-E3(3)-E3(2)-E11(1)-E4(1)- } \\
\text { D2(0) }\end{array}$ & 7 & 4.4 & $\begin{array}{l}\text { D2(0)-S3(1)-S6(4)-S5(10)- } \\
\text { E25(9)-E26(8)-E24(6)- } \\
\text { E23(5)-E10(4)-E6(3)-E4(2)- } \\
\text { E9(0)-D2(0) }\end{array}$ & 8 & 10.6 \\
\hline 2 & $\begin{array}{l}\text { D3(0)-S1(5)-E14(4)-E13(3)-E12(1)- } \\
\text { E8(0)-S2(5)-E7(3)-E19(2)-E22(0)- } \\
\text { D3(0) }\end{array}$ & 7 & 6.2 & $\begin{array}{l}\text { D3(0)-S2(3)-S6(5)-S3(101)- } \\
\text { E5(8)-E3(7)-E2(6)-E2(5)- } \\
\text { E3(3)-E11(2)-E7(0)-D3(0) }\end{array}$ & 7 & 7.2 \\
\hline 3 & $\begin{array}{l}\text { D3(0)-S2(3)-E2(2)-E15(0)-S3(1)- } \\
\text { E6(0)-S5(6)-E25(5)-E26(4)-E24(2)- } \\
\text { E23(1)-E10(1)-D3(0) }\end{array}$ & 8 & 13.3 & $\begin{array}{l}\text { D3(0)-S1(3)-S2(4)-S4(10)- } \\
\text { E29(9)-E28(8)-E19(7)- } \\
\text { E22(5)-E18(4)-E21(3)- } \\
\text { E17(2)-E14(1)-E13(0)-D3(0) }\end{array}$ & 9 & 10.1 \\
\hline 4 & $\begin{array}{l}\text { D1(0)-S4(8)-E29(7)-E1(6)-S2(7)- } \\
\text { E18(6)-S1(7)-E17(6)-E21(5)-E28(4)- } \\
\text { E27(1)-E16(0)-D1(0) }\end{array}$ & 8 & 10.2 & $\begin{array}{l}\mathrm{D} 1(0)-\mathrm{S} 4(5)-\mathrm{S} 2(7)-\mathrm{S} 1(10)- \\
\text { E12(8)-E15(6)-E8(5)-E1(4)- } \\
\text { E16(3)-E27(0)-D1(0) }\end{array}$ & 6 & 8.4 \\
\hline
\end{tabular}

\subsection{Parameter sensitivity analysis}

The effect of the number of distribution centers on the mileage distribution via the ILU and LBU modes was also analyzed in detail. According to the data in Table 2, the following conclusions were made.

(1) The total distributed mileage gradually increased as the number of distribution centers decreased, because the UAVs could not depart from the nearest distribution center, which increased the mileage.

(2) The total distributed mileage of the ILU was less than that of LBU, because the latter mode implied visiting the collection point first and then the delivery point, discarding a part of the return path to the distribution center.

Table 2. The effect of the number of dispatch centers on scheduling results

\begin{tabular}{|c|c|c|c|c|c|c|}
\hline \multirow{2}{*}{$\begin{array}{l}\text { Number of } \\
\text { dispatch } \\
\text { centers }\end{array}$} & \multicolumn{3}{|c|}{$\begin{array}{l}\text { Integrated loading and unloading } \\
\text { (ILU) }\end{array}$} & \multicolumn{3}{|c|}{ Loading before unloading (LBU) } \\
\hline & $\begin{array}{l}\text { Number } \\
\text { of UAVs }\end{array}$ & $\begin{array}{l}\text { Dispatch } \\
\text { centers }\end{array}$ & $\begin{array}{l}\text { Total } \\
\text { mileage } \\
(\mathrm{km})\end{array}$ & $\begin{array}{l}\text { Number } \\
\text { of UAVs }\end{array}$ & $\begin{array}{l}\text { Dispatch } \\
\text { centers }\end{array}$ & $\begin{array}{l}\text { Total mileage } \\
(\mathrm{km})\end{array}$ \\
\hline 1 & 5 & D2 & 37.1 & 4 & D2 & 41.1 \\
\hline 2 & 4 & D2, D3 & 35.0 & 4 & D2, D3 & 37.0 \\
\hline 3 & 4 & $\mathrm{D} 1, \mathrm{D} 2, \mathrm{D} 3$ & 34.1 & 4 & $\mathrm{D} 1, \mathrm{D} 2, \mathrm{D} 3$ & 36.3 \\
\hline
\end{tabular}


The effect of applying several UAVs types with varying maximum mileages and cargo weights via the ILU and LBU modes on the delivery efficiency was also analyzed. The results are summarized in Table 3.

Table 3. The effect of different drone types on scheduling results

\begin{tabular}{llllllll}
\hline UAV types & \multicolumn{3}{c}{$\begin{array}{l}\text { Integrated loading and unloading } \\
\text { (ILU) }\end{array}$} & \multicolumn{2}{l}{ Loading before unloading (LBU) } \\
$\begin{array}{l}\text { Weight } \\
\text { (kg) }\end{array}$ & $\begin{array}{l}\text { Mileage } \\
(\mathbf{k m})\end{array}$ & $\begin{array}{l}\text { Number } \\
\text { of } \\
\text { UAVs }\end{array}$ & $\begin{array}{l}\text { Dispatch } \\
\text { center }\end{array}$ & $\begin{array}{l}\text { Total } \\
\text { mileage } \\
(\mathbf{k m})\end{array}$ & $\begin{array}{l}\text { Number } \\
\text { of } \\
\text { UAVs }\end{array}$ & $\begin{array}{l}\text { Dispatch } \\
\text { center }\end{array}$ & $\begin{array}{l}\text { Total } \\
\text { mileage } \\
(\mathbf{k m})\end{array}$ \\
\hline 10 & 50 & 4 & D1, D2, D3 & 34.1 & 4 & D1, D2, D3 & 36.3 \\
10 & 20 & 5 & D1, D2, D3 & 34.3 & 5 & D1, D3 & 37.1 \\
20 & 20 & 2 & D2, D3 & 29.6 & 2 & D1,D3 & 32.1 \\
\hline
\end{tabular}

It can be concluded from results in Table 3 that:

(1) for the UAVs with the same cargo weight limit, when their maximum mileage decreases, more UAVs are needed to fulfill the same number of orders, and the total delivery mileage grows because the return to the distribution center increases the invalid mileage

(2) for the UAVs with the same maximum mileage limit, when their maximum load decreases, more UAVs are also needed to fulfill the same number of orders. Besides, the differences between the ILU and LBU scheduling schemes are consistent with the above analysis.

In addition, the impact of different order sizes on the computation time was analyzed, as shown in Table 4.

Table 4. The effect of different order sizes on computation time

\begin{tabular}{|c|c|c|}
\hline Number of orders & $\begin{array}{l}\text { Integrated loading and unloading } \\
\text { (ILU) } \\
\text { Computation time(s) }\end{array}$ & $\begin{array}{l}\text { Loading before unloading (LBU) } \\
\text { Computation time(s) }\end{array}$ \\
\hline 30 & 195.459 & 221.921 \\
\hline 60 & 251.237 & 394.518 \\
\hline 120 & 719.255 & 1099.798 \\
\hline
\end{tabular}

As seen in Table 4, the computation time increased gradually with the problem scale. However, it was within the acceptable time range, thus proving the proposed model and algorithm effectiveness. Besides, the ILU total computation time was less than the LBU one, since the latter implied the increased attribute discrimination operation time of some nodes by traversing the loading and unloading separately. The computation results are in line with the intuitive analysis.

\section{Conclusions}

In this study, the ant colony optimization (ACO) algorithm was used to solve the UAV distribution path optimization model with an integrated loading and unloading (ILU), taking into account the no-fly zone that increased the flight mileage and UAVs' performance origin and destination of customer orders, and other realistic constraints. Comparing the ILU and LBU (loading before unloading) distribution modes, the relationships between the number of UAVs in distribution centers, distribution routes, and transportation costs were identified. The results show that the ILU mode can effectively reduce transportation mileage, make full use of UAVs for pick-up and delivery services, and improve the efficiency of UAVs. Finally, the GIS-based distribution platform using Java Development Kit was elaborated and tested. The following conclusions were drawn: 
(1) The proposed model could effectively integrate the collection and delivery tasks and calculate the best scheduling plans for the ILU and LBU modes. The parameter sensitivity analysis for the multipoint pick-up and delivery by UAV was performed. The total transportation mileage of the ILU mode was systematically less than that of LBU, which is considered instrumental in drone delivery optimization practice.

(2) The heuristic algorithm designed in this paper successfully found feasible solutions to the large-scale problem in an acceptable time.

However, this paper addressed the logistics distribution problem for a single type of UAVs and cargo. In reality, the types of UAVs and the diversity of items in the distribution demand greatly impact the distribution scheme. The interrelations among the above factors are vital in finding the best resource allocation, which will be our future research direction.

\section{Acknowledgements:}

This paper is funded by the Central College Basic Scientific Research Operating Expenses Fund in Civil Aviation University of China 3122019126.

\section{References}

[1] Murray C C, Chu A G. The flying sidekick traveling salesman problem: Optimization of drone-assisted parcel delivery. Transportation Research Part C Emerging Technologies, 2015, 54(5): 86-109.

[2] Agatz N, Bouman P, Schmidt M. Optimization approaches for the traveling salesman problem with drone. Transportation Science, 2018, 52(4): 965-981.

[3] Ha Q M, Deville Y, Pham Q D, et al. On the min-cost traveling salesman problem with drone. Transportation Research Part C, 2018, 86: 597-621.

[4] Marinelli M, Caggiani L, Ottomanelli M, et al. En route truck drone parcel delivery for optimal vehicle routing strategies. IET Intelligent Transport Systems, 2018, 12(4):253-261.

[5] Wang K, Yuan B, Zhao M, et al. Cooperative route planning for the drone and truck in delivery services: A bi-objective optimisation approach. Journal of the Operational Research Society, 2019, 5:1-18.

[6] Ferrandez S M, Harbison T, Weber T, et al. Optimization of a truck-drone in tandem delivery network using K-means and genetic algorithm. Journal of Industrial Engineering and Management,2016,9(2):374-388.

[7] Chang Y S, Lee H J. Optimal delivery routing with wider drone delivery areas along a shorter truck route. Expert Systems with Applications, 2018, 104:307-317.

[8] Ham Andy M. Integrated scheduling of m-truck, m-drone, and m-depot constrained by time-window, drop-pickup, and m-visit using constraint programming. Transportation Research Part C, 2018, 91(4): $1-14$.

[9] González P, Canca D, Andrade J, et al. Truck-drone team logistics: A heuristic approach to multi-drop route planning. Transportation Research Part C, 2020, 114(7): 657-680.

[10] Dorling K, Heinrichs J, Messier G G, et al. Vehicle routing problems for drone delivery. IEEE Transactions on Systems Man \& Cybernetics Systems, 2017, 47(1): 70-85.

[11] Rabta B, Wankmüller C, Reiner G.A Drone fleet model for lastmile distribution in disaster relief operations. International Journal of Disaster Risk Reduction, 2018, 28:107-112.

[12] Wen T, Zhang Z, Wong K K L. Multi-objective algorithm for blood supply via unmanned aerial vehicles to the wounded in an emergency situation. Plos One, 2016,11(5):1-22.

[13] Kitjacharoenchai P, Min B C, Lee S. Two echelon vehicle routing problem with drones in last-mile delivery. International Journal of Production Economics, 2020, 225(12): 1-14.

[14] Salama M, Srinivas S. Joint optimization of customer location clustering and drone-based routing for last-mile deliveries. Transportation Research Part C, 2020, 114(7): 620-642.

[15] Tang L, Hao P, Zhang X J. Path planning method for mountain UAV Based on improved ant colony algorithm. Transportation system engineering and information, 2019, 19 (1): 158-164.

[16] Song A, Bao X Z. Elite diffusion ant colony algorithm for three-dimensional path planning of transportation drones. Computer Engineering and Science, 2020. 